\title{
CONSTRUCCION Y UTILIZACION DE UN ELECTRODIALIZADOR EN SERIE PARA LABORATORIO
}

\author{
Antonio Montes-Rojas* y Luis Manuel Álvarez Cerda
}

Facultad de Ciencias Químicas, Universidad Autónoma de San Luis Potosí, CP 78250, San Luis Potosí, S.L.P. México

Recebido em 31/3/08; aceito em 21/8/08; publicado na web em 2/2/09

\begin{abstract}
MANUFACTURING AND USING OF AN ELECTRODIALYZER IN SERIES TO LABORATORY. There are different concepts in electrochemistry that must be supported by a laboratory practice to better understand their importance in industrial applications. This work reports the procedure for manufacturing an electrodialyzer in series of two or more compartments with easy manipulation. Likewise, it reports the results obtained by using this system in the nitrate elimination from synthetic $\mathrm{NaNO}_{3}$ solutions at different concentrations. Among other results obtained in the electrodialysis of these solutions, the one that stands out is the demonstration of the linear relationship between the limiting current, obtained from the polarization curves, and nitrate concentration. Additionally, implementation of the separation treatment shows that the time of elimination of practically all ionic species depends on their concentration in solution and ranges between 50 and $80 \mathrm{~min}$.
\end{abstract}

Keywords: electrodialysis; electrodialyzer; nitrates.

\section{INTRODUCCION}

La electrodiálisis es un proceso de separación asistido con membranas selectivas que involucra la utilización de diferentes conceptos como el número de transporte, la movilidad o el transporte por migración.

Esta metodología ha sido utilizada de manera exitosa en la desalación de agua de mar más en los últimos años su utilización ha abarcado el tratamiento de aguas residuales. ${ }^{1,2}$ Entre las ventajas que posee la electrodiálisis es que su implementación experimental se lleva a cabo en condiciones suaves, o bien el uso de agentes químicos durante el desarrollo del proceso de separación se reduce a lo mínimo.

No obstante, se debe mencionar que existen muy pocos trabajos ${ }^{3-6}$ que han resaltado la importancia de este tipo de procesos en la enseñanza a nivel licenciatura de la ingeniería química o afines, por lo que la comunidad docente parece mantener un desinterés en incluirlos en los programas de estudios. Kendall ${ }^{3}$ y colaboradores exponen tres razones por las cuales se cree que los procesos de membranas o la electrodiálisis se mantiene excluida de estos últimos: 1) su tratamiento en los libros de texto es inadecuada para el nivel de licenciatura, 2) sus ventajas y aplicaciones no son completamente entendidas y 3) la aplicación en el laboratorio no es barata. Cabe señalar con relación a la razón número 3 , que García-García ${ }^{4}$ y colaboradores han propuesto la realización de un dispositivo de electrodiálisis de bajo costo para la separación de un aminoácido a partir de una solución acuosa en lugar de una separación por precipitación en fase orgánica. Este trabajo se caracteriza por introducir al estudiante de manera breve a los principios de la electrodiálisis; sin embargo, el desarrollo experimental es demasiado largo por lo que dificulta su realización en una sola sesión.

Es así, que con el propósito contribuir a una mejor comprensión de la importancia de la electrodiálisis, se propone la construcción de un dispositivo de electrodiálisis de fácil manipulación y muy económico. Adicionalmente, este dispositivo se utiliza en la separación de iones nitrato presentes en una solución sintética, especie que posee un interés importante desde el punto de vista de la remediación ambiental. ${ }^{7,8}$

*e-mail: antonio.montes@uaslp.mx

\section{SECCION TEORICA}

\section{Membranas selectivas}

Uno de los componentes importantes de la electrodiálisis es la membrana que se utiliza para efectuar la separación. Esta membrana posee propiedades selectivas y puede ser considerada ${ }^{1}$ como una barrera que separa dos fases y que bajo el efecto de un campo eléctrico, $\Delta \psi$, formado por la aplicación de una diferencia de potencial entre dos electrodos, ciertos componentes de la fase podrán atravesarla pero otros serán repelidos.

Para que este proceso de separación se produzca, en la membrana existen diferentes elementos como los grupos funcionales fijos cargados que determinan la permeabilidad de las mismas según el signo que posean, Figura 1. Por ejemplo, si la membrana posee cargas fijas positivas estas dejaran pasar solamente a las especies de signo opuesto en razón a la atracción electrostática que existe entre ellas; por otra parte, las especies que tengan el mismo signo serán repelidas.

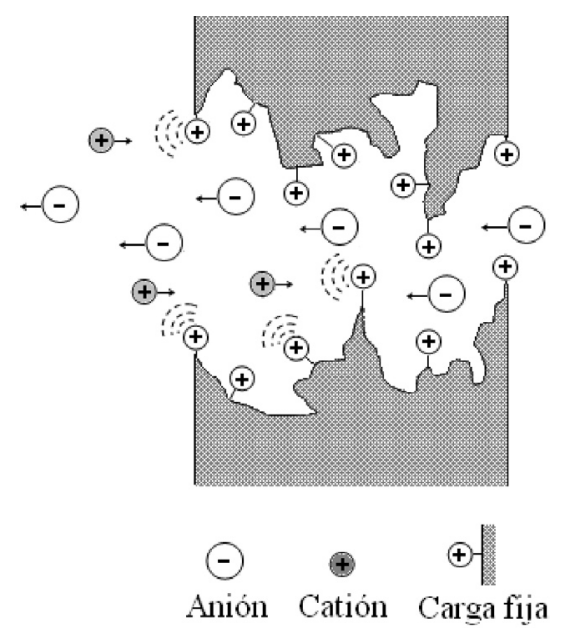

Figura 1. Esquema de una membrana selectiva de intercambio aniónico donde se pueden apreciar las interacciones entre las diferentes especies cargadas: cargas fijas, contraiónes (anión) y co-iones (catión) 
En la estructura de la membrana existen especies iónicas móviles que pueden desplazarse tanto en ella como en la solución y reciben el nombre de contraiones, debido a que poseen una carga de signo opuesto a la de las cargas fijas. Estas especies dan electroneutralidad a las membranas y son sustituidas por los iones que se desplazan a través de ellas cuando inicia el proceso de separación. A su vez, los iones móviles pero de igual signo que las cargas fijas en la membrana, reciben el nombre de co-iones.

En el caso en que las membranas estén compuestas de cargas fijas de signo negativo, entonces permitirán el paso de especies sólo de carga positiva.

\section{Proceso de transporte a través de una membrana selectiva}

Uno de los factores que influye fuertemente sobre el proceso de transporte iónico a través de membranas es el que esta relacionado con el que una membrana separe a dos fases, que en algunos casos pueden ser de concentración muy diferente. Esto es, cuando la corriente eléctrica inducida por la diferencia de potencial pasa a través de un sistema que contiene una membrana, la corriente fluye en la solución por medio de los cationes y los aniones presentes en ella, mientras que en la membrana fluye principalmente por medio de los contraiones. La diferencia entre las velocidades con las que se desplazan (movilidad) estos en la membrana y en la solución conduce a una diferencia en la concentración de cada uno de ellos en una delgada capa $(\delta)$ en las proximidades de la membrana.

Si la diferencia de potencial aumenta, entonces la densidad de corriente debe aumentar, la densidad del flujo va aumentar y consecuentemente la concentración del contraión va a disminuir (compartimiento diluido). Cuando la concentración del contraión en la superficie de la membrana $\mathrm{c}_{m}$ se aproxima a cero se presenta una corriente límite, $\mathrm{i}_{\text {lim }}$, dada por la Ecuación 1 para el caso de una membrana intercambiadora de cationes:

$$
i_{\lim }=\frac{z D F c_{b}}{\delta\left(t^{m}-t^{b l}\right)}
$$

Donde $\mathrm{t}^{\mathrm{m}} \mathrm{y} \mathrm{t}^{\mathrm{bl}}$ son el número de transporte del contraión en la membrana y en la solución, $\mathrm{z}$ es la carga de la especie iónica, F es la constante de Faraday (96495 $\mathrm{C} \mathrm{mol}^{-1}$ ), D es el coeficiente de difusión $\mathrm{y} \mathrm{c}_{\mathrm{b}}$ es la concentración del contraión en el seno de la solución.

Un incremento adicional en la fuerza impulsora (por aumento del potencial eléctrico) no va a resultar en un aumento del flujo del catión de interés sino de los iones $\mathrm{H}^{+}$y $\mathrm{OH}^{-}$del agua formados justo en ese momento. Según la expresión 1, se puede ver que la densidad de corriente límite depende de la concentración del contraión (catión) en el seno de la solución $\mathrm{c}_{b}$ y del espesor de la capa próxima a la membrana. Cabe señalar que estos dos parámetros son importantes para definir las condiciones de operación de un electrodializador cuando estos procesos de membranas son utilizados para la separación de especies iónicas.

Si bien se ha descrito el efecto de la polarización con los cationes, el mismo análisis es valido para el caso de los aniones, aunque se debe considerar que la movilidad de los aniones, con una misma valencia en la capa próxima a la membrana es menos importante que la de los cationes.

Es importante mencionar que los procesos anteriores presentes en el transporte iónico de una membrana pueden describirse con la ayuda de una curva de polarización, la cual es la representación gráfica de la intensidad de corriente en función de la diferencia de potencial desarrollada en la proximidad de una membrana durante el transporte iónico a través de ella. En la sección Material Suplementar se describe con mayor detalle este tipo de curvas, así como la forma en que se puede obtener algunos parámetros de operación como la corriente límite.

\section{Separación de componentes iónicos utilizando la electrodiálisis}

Si bien es interesante desde el punto de vista fundamental estudiar los procesos que ocurren al tener una sola membrana, su aplicación práctica implica un arreglo alternado de varias membranas selectivas. Este proceso se conoce formalmente como electrodiálisis y permite no solamente la separación de componentes iónicos presentes en una fase sino también la obtención de ciertos productos. ${ }^{9-11}$

En la electrodiálisis la estructura experimental que contiene tanto a las membranas selectivas como a los electrodos da lugar a la formación de compartimientos a través de los cuales se puede alimentar el efluente a tratar, Figura 2. Durante el desarrollo del proceso, el trasporte iónico a través de la membrana da lugar a la formación de canales a través de los cuales se producen dos tipos de efluentes: un diluido en la concentración de la especie de interés y un concentrado en la misma. Esto se lleva a cabo de la siguiente forma:

Al tener un arreglo alternado de membranas de intercambio iónico (catiónica y aniónica), las especies iónicas trataran de migrar hacia su respectivo electrodo por la imposición del campo eléctrico o por una corriente, por ejemplo, las especies cargadas negativamente migrarán hacia el ánodo mientras que las especies cargadas positivamente migrarán hacia el cátodo, esto ocurrirá si es que no existe algo que les impida este transporte direccional. Si se tiene la disposición alternada como se aprecia en la Figura 2, en el compartimiento de la derecha a la membrana aniónica (MA) ocurrirá un empobrecimiento de los iónes; mientras que, en el compartimiento de la izquierda, se concentrarán las especies iónicas en solución para así, obtener dos tipos de corriente producto: una diluida y otra concentrada de la especie a tratar.

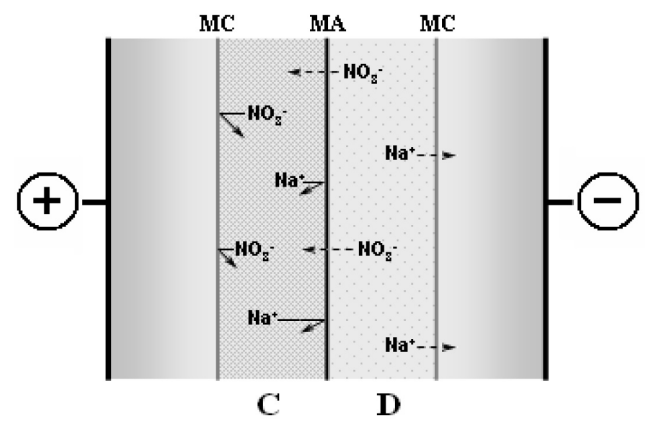

Figura 2. Funcionamiento del proceso de múltiples membranas (MC YMA) en el que se forman dos tipos de efluentes: uno diluido $(D)$ y otro concentrado $(C)$

Es importante considerar que la alimentación de la solución a tratar a través del electrodializador puede hacerse de dos formas: a) Modo en serie - la solución recorre sucesivamente cada uno de los diferentes compartimientos que le son reservados y que están comunicados entre sí, lo que permite obtener más rápidamente la concentración buscada; b) Modo en paralelo - la solución circula una sola vez en los diversos compartimientos independientes que le son reservados en el electrodializador.

\section{PARTE EXPERIMENTAL}

\section{Soluciones}

Todas las soluciones utilizadas en este trabajo fueron preparadas a partir de sustancias grado reactivo y agua desionizada con una conductividad de $18 \mathrm{M} \Omega \mathrm{cm}$. 


\section{Montaje experimental}

El proceso de electrodiálisis se realizó con flujo utilizando el siguiente montaje experimental, Figura 3.

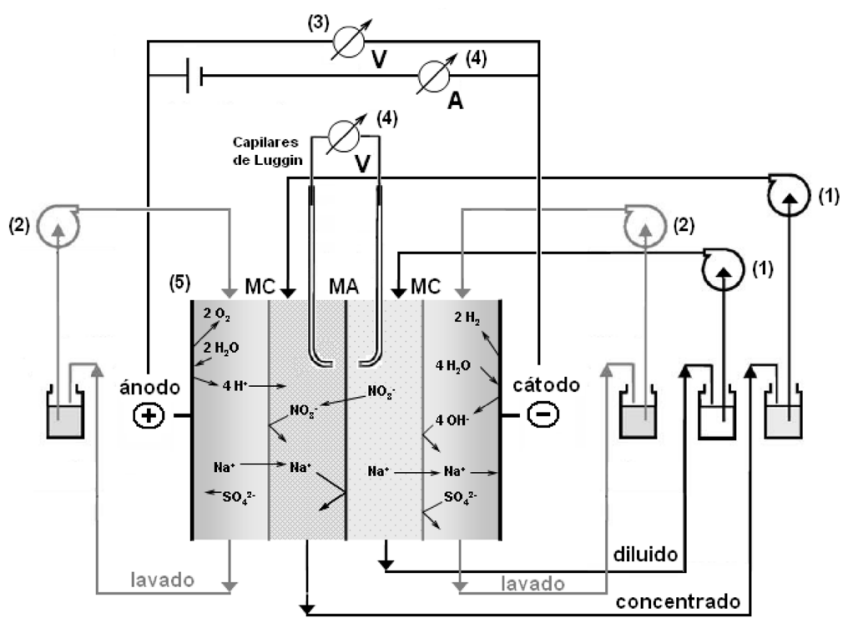

Figura 3. Diagrama del sistema experimental de electrodiálisis con dos compartimientos; membrana de intercambio aniónico (MA), membrana de intercambio catiónico (MC), 1) bombas peristálticas, 2) bombas tipo fuente, 3) fuente de poder, 4) multímetros y 5) electrodializador

Dicho montaje incluyó a dos tipos de elementos: eléctricos - una fuente de poder para imponer la corriente de tratamiento, un multímetro para leer la corriente que fluye en el electrodializador, un multímetro para leer la diferencia de potencial desarrollada durante el transporte iónico en la membrana, dos celdas de $\mathrm{Ag} \mathrm{AgCl} 3 \mathrm{M}$ $\mathrm{NaCl}$ (BAS) como electrodos de referencia para detectar la diferencia de potencial de la membrana; hidráulicos - una bomba tipo fuente para cada uno de los compartimientos que alojan a los electrodos, dos bombas peristálticas para modular el flujo de la solución de tratamiento.

\section{Electrodializador en serie}

El electrodializador utilizado en este trabajo fue construido en el laboratorio de modo a que la solución circule en serie, a partir de cortes de una hoja de acrílico de espesor de $5 \mathrm{~mm}$ para obtener dos tipos de componentes: a) Componente que aloja a cada electrodo y que le confiere hermeticidad al electrodializador, Figura 4A.

Este componente se preparó por duplicado ensamblando dos elementos de diferentes dimensiones: El primero es una lamina continua de 2 x 16 x $19 \mathrm{~cm}$ que junto con su contraparte mantienen unidos a todos los elementos del electrodializador con tornillos largos de calibre de 1/4 de pulgada de longitud dependiente del numero de compartimientos, los cuales se colocan en los bordes de cada lado del componente a manera de un reactor filtro-prensa, como se ilustra en sólo uno de los lados de la representación en la Figura 4A.

El otro componente es un rectángulo de $1 \times 12 \times 15 \mathrm{~cm}$ con un hueco de $1 \times 7 \times 11 \mathrm{~cm}$, el cual aloja a la malla metálica que sirve como electrodo y que a su vez permite la circulación de la solución de lavado de los electrodos. Para cumplir esta doble función el componente presenta un orificio en uno de los lados por el que se puede introducir un alambre para el contacto eléctrico entre el electrodo y la fuente de poder; mientras que del lado opuesto, dos orificios permiten la circulación de la solución de lavado.

Cabe señalar que los dos elementos anteriores pueden unirse
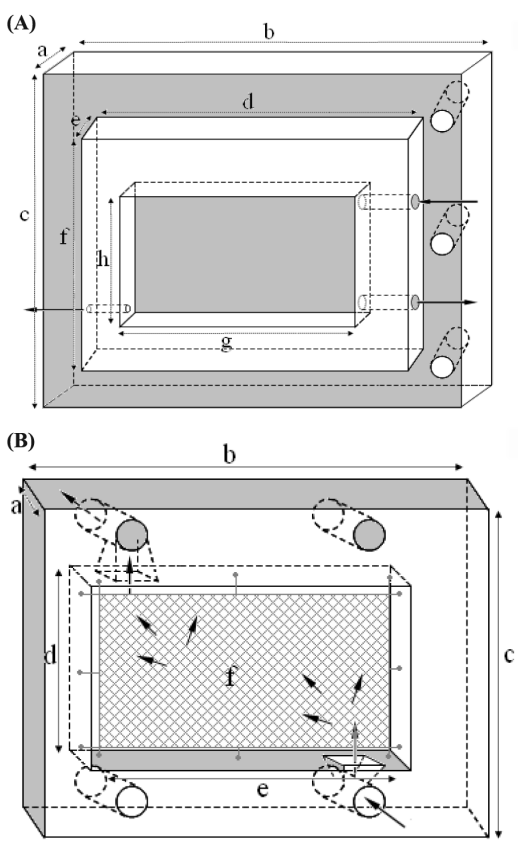

Figura 4. (A) Componente que aloja a cada electrodo y que permite la hermeticidad al electrodializador. Las dimensiones del componente son: $a=2$ $\mathrm{cm}, b=19 \mathrm{~cm}, c=16 \mathrm{~cm}, d=15 \mathrm{~cm}, e=1 \mathrm{~cm}, f=12 \mathrm{~cm}, \mathrm{~g}=11 \mathrm{~cm}, h=7$ $\mathrm{cm}$. (B) Componente entre membranas que permite la disposición alternada de las membranas selectivas y el flujo en serie del efluente a través del electrodializador. Las dimensiones del componente son: $a=0.5 \mathrm{~cm}, b=15 \mathrm{~cm}$, $c=12 \mathrm{~cm}, d=7 \mathrm{~cm}, e=11 \mathrm{~cm}, e:$ malla plástica

permanentemente con cloroformo o de manera temporal con algún adhesivo; además, para alcanzar el espesor requerido se puede lograr con cortes simétricos sucesivos de la lamina de $0.5 \mathrm{~cm}$ o de un solo corte de una lamina de mayor espesor (¡más difícil!), lo cual implica una mayor habilidad para hacerlo.

b) Componentes entre membranas, Figura 4B. Este componente funciona como una plantilla en el electrodializador y permite ensamblar las membranas alternadamente en cada una de sus caras, por lo que el número de ellos depende del número de compartimientos deseados. La forma en como se construyó fue con un corte de la lamina de acrílico de $0.5 \mathrm{~cm}$ de espesor (o más delgada) y de 12 x $15 \mathrm{~cm}$. Este elemento presenta un hueco en el centro de 7 x $11 \mathrm{~cm}$ que permite la circulación de la solución a tratar con 4 orificios de $1 \mathrm{~cm}$ de diámetro y que gracias a una malla de plástico provoca turbulencias en ella para una mejor eficiencia en el transporte iónico.

Con el propósito de mantener separados los dos tipos de productos generados durante el tratamiento de electrodiálisis, esta plantilla presenta en dos de los orificios un hueco paralelo por el que circula exclusivamente sólo un tipo de producto. Estos componentes junto con los electrodos se ensamblaron como se ilustra en la Figura 5.

El ensamble de los componentes anteriormente descritos permite la obtención del electrodializador y debe comprobarse que en este no existan fugas o que se mezclen los productos.

\section{Electrodos}

Los electrodos utilizados en este trabajo fueron dos mallas de platino con alambres de $1 \mathrm{~mm}$ de diámetro y con dimensiones de 6 x $10 \mathrm{~cm}$. Si bien estos son una parte costosa del electrodializador, se puede reducir el costo de ellos al utilizar sólo uno para el ánodo o ambos de acero inoxidable. 


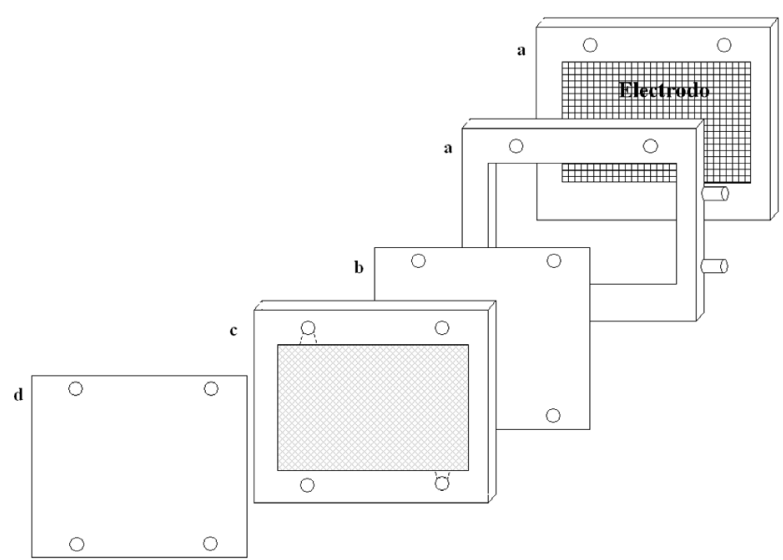

Figura 5. Ensamble de los componentes para el electrodializador. a) Componente para alojar a los electrodos (en este caso están separados los dos elementos que lo constituyen), b) membrana catiónica, c) componente entre membranas, d) membrana de intercambio aniónico, y así sucesivamente hasta tener los compartimiento deseados

\section{Membranas y su tratamiento}

Las membranas utilizadas (Tokuyama) fueron dos cortes de membranas catiónicas (CMX) y una membrana aniónica (ACS), con el objeto de obtener un compartimiento de diluido y otro de concentrado, Figura 3.

Antes de cada experimento se estabilizaron las membranas sumergiéndolas en una solución de nitrato de sodio de la misma concentración que de la solución a tratar durante 12 h.

\section{Solución de lavado de los electrodos}

Para preparar la solución de lavado de los electrodos se utilizó $\mathrm{Na}_{2} \mathrm{SO}_{4}$ grado reactivo (Fermont) en una concentración de 0,3 M.

\section{RESULTADOS Y DISCUSION}

\section{Condiciones de operación del tratamiento de electrodiálisis}

A partir de las curvas de polarización se determinó la corriente límite a través del método de Cowan y Brown ${ }^{12}$ para todo el intervalo de

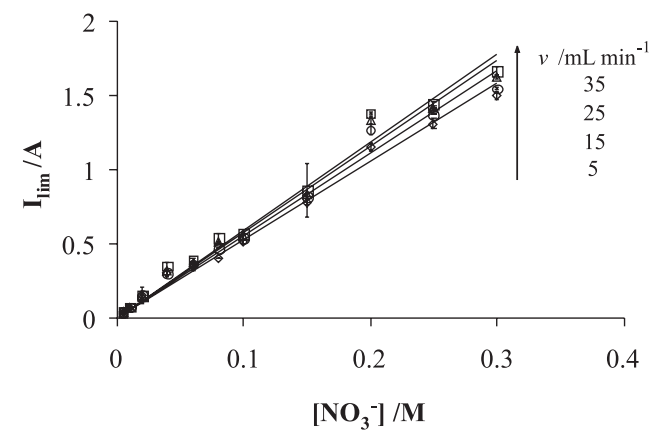

Figura 6. Curvas de la corriente límite ( $I_{\text {lim }}$ ) en función de la concentración

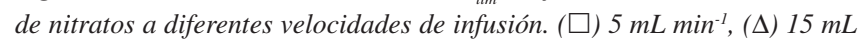
$\mathrm{min}^{-1}$, (○) $25 \mathrm{~mL} \mathrm{~min}{ }^{-1},(\diamond) 35 \mathrm{~mL} \mathrm{~min}{ }^{-1}$

concentraciones de nitratos estudiado utilizando diferentes velocidades de flujo. Los resultados obtenidos se muestran en la Figura 6.

Tal y como se mencionó en la sección teórica, la corriente límite aumenta a medida que lo hace la concentración de nitrato puesto que a mayor concentración de especie iónica es mayor el flujo ( $\left.\mathrm{I}_{\lim }\right)$ de ésta a través de la membrana. Adicionalmente, si la velocidad de alimentación ( $v$ ) aumenta, el espesor disminuye en razón a que un mayor flujo se elimina parte del gradiente de concentración formado por el trasporte del contraión. Es así que este comportamiento de la curva $\mathrm{I}_{\lim } v s\left[\mathrm{NO}_{3}^{-}\right]$refleja la validez de la Ecuación 1.

En la Tabla 1 se presentan todos los valores de la corriente límite obtenidos que se utilizaron para preparar la curvas de la Figura 6.

Según estos valores se confirma el buen funcionamiento del electrodializador, y por tanto nos permite pasar al proceso de electrodiálisis de las soluciones de nitrato.

\section{Electrodiálisis de las soluciones de nitrato}

La eficiencia del proceso de electrodiálisis se determinó con las curvas del porcentaje de remoción (\%R), para todo el rango de velocidades de infusión estudiado, en función de la concentración inicial de nitratos, $\left[\mathrm{NO}_{3}^{-}\right]$, a un tiempo de tratamiento de 41 min, Figura 7.

De acuerdo con estas curvas, la eficiencia a la remoción de nitratos utilizando esta membrana varía entre 40 y $~ 80 \%$ si la concentración de especie iónica es menor a $0.1 \mathrm{M}$ manteniendo constante la velocidad de alimentación $(v)$. Mas si la concentración de iones nitrato

Tabla 1. Corrientes límite $\left(\mathrm{I}_{\mathrm{lim}}\right)$ obtenidas a partir de las curvas de polarización en todo el rango de concentraciones y velocidades $(v)$ en estudio para la membrana de intercambio aniónico ACS

$$
\mathrm{I}_{\lim } / \mathrm{A}
$$

\begin{tabular}{lccccccc}
{$\left[\mathrm{NO}_{3}^{-}\right] / \mathrm{M}$} & $0 \mathrm{~mL} \mathrm{~min}^{-1}$ & $2.5 \mathrm{~mL} \mathrm{~min}^{-1}$ & $5 \mathrm{~mL} \mathrm{~min}^{-1}$ & $10 \mathrm{~mL} \mathrm{~min}^{-1}$ & $15 \mathrm{~mL} \mathrm{~min}^{-1}$ & $25 \mathrm{~mL} \mathrm{~min}^{-1}$ & $35 \mathrm{~mL} \mathrm{~min}^{-1}$ \\
\hline 0.3 & 1.430 & 1.474 & 1.501 & 1.518 & 1.548 & 1.624 & 1.670 \\
0.25 & 1.241 & 1.267 & 1.312 & 1.347 & 1.375 & 1.420 & 1.440 \\
0.2 & 1.101 & 1.125 & 1.156 & 1.182 & 1.270 & 1.330 & 1.381 \\
0.15 & 0.731 & 0.766 & 0.783 & 0.803 & 0.820 & 0.828 & 0.863 \\
0.1 & 0.495 & 0.512 & 0.518 & 0.532 & 0.542 & 0.562 & 0.566 \\
0.08 & 0.360 & 0.388 & 0.404 & 0.415 & 0.476 & 0.512 & 0.544 \\
0.06 & 0.304 & 0.337 & 0.350 & 0.354 & 0.367 & 0.376 & 0.393 \\
0.04 & 0.281 & 0.290 & 0.299 & 0.302 & 0.307 & 0.325 & 0.353 \\
0.02 & 0.140 & 0.145 & 0.148 & 0.148 & 0.152 & 0.153 & 0.156 \\
0.01 & 0.070 & 0.072 & 0.073 & 0.074 & 0.076 & 0.076 & 0.076 \\
0.005 & 0.035 & 0.036 & 0.036 & 0.037 & 0.038 & 0.038 & 0.038 \\
\hline
\end{tabular}




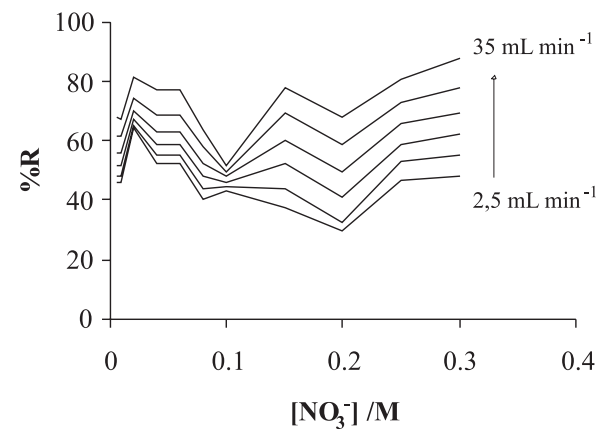

Figura 7. Porcentajes de remoción (\%R) en función de la concentración del efluente [ $\left.\mathrm{NO}_{3}^{-}\right]$a diferentes velocidades de infusión para las membrana ACS a $0,8 I_{\text {lim }}$

es mayor a $0.1 \mathrm{M}$, entonces este mismo parámetro varía entre 30 y $70 \%$ para un mismo valor de $v$. Este comportamiento de $\% \mathrm{R}$ parece estar asociado tanto a la membrana como a la concentración del ión nitrato más no al diseño del electrodializador. En este sentido, creemos que a concentraciones de nitratos inferiores a $0.1 \mathrm{M}$, la separación de los aniones se ve favorecida prioritariamente por las propiedades selectivas de la membrana y que estas propiedades se van perdiendo conforme la concentración de nitratos se aproxima a $0.1 \mathrm{M}$, sin importar el valor de la velocidad de alimentación. Por otra parte a concentraciones de nitratos superiores a $0.1 \mathrm{M}$, la velocidad de alimentación es más importante que las propiedades selectivas de la membrana y por lo tanto favorece la separación de los aniones conforme $v$ aumenta.

\section{CONCLUSIONES}

Las conclusiones que se pueden mencionar son básicamente tres: el dispositivo experimental utilizado es de fácil manejo, lo que permite realizar diferentes experimentos en sólo una sesión de laboratorio de aproximadamente $3 \mathrm{~h}$; los resultados obtenidos con la celda diseñada se ajustan a la teoría desarrolla en la literatura con lo cual se pueden proponer el calculo de diferentes parámetros para evaluar el funcionamiento del transporte de iones a través de una membrana aniónica. Este tratamiento de los resultados puede hacerse fuera del tiempo de práctica, por ejemplo para preparar el reporte escrito de la misma; la eliminación de los iones nitratos por electrodiálisis esta influenciada tanto por las propiedades selectivas de la membrana, la velocidad de alimentación de la solución como de la concentración de los aniones. Según nuestros resultados, la remoción de los aniones se produce entre 50 y 80 min para un amplio rango de concentraciones.

\section{Perspectivas de aplicación en laboratorio}

Uno de los aspectos importantes que se debe de considerar para incluir este tipo de experimentos en el manual de prácticas de licenciaturas afines a la química, es la facilidad para ensamblar el dispositivo así como en el reducido costo que implica construirlo. Sin embargo, se debe tomar en cuenta que el uso de algunos elementos utilizados en este trabajo, como el material de los electrodos, pueden sustituirse a fin de que los costos disminuyan aun más.

\section{MATERIAL SUPLEMENTAR}

Disponible en http://quimicanova.sbq.org.br, en arquivo PDF.

\section{AGRADECIMIENTOS}

A la empresa Thyssenkrupp-Mexinox S.A. de C.V. y a la Universidad Autónoma de San Luis Potosí a través del Fondo de Apoyo a la Docencia FAD0310QBS/38. LMAC agradece a CONACYT la beca para realizar estudios de maestría. Los autores agradecen igualmente a L. Q. A. E. R. Olmos por su contribución a este trabajo así como a la Dra. L. M. T. Rodríguez por sus comentarios sobre la redacción de este trabajo.

\section{REFERENCIAS}

1. Mulder, M.; Basic principles of membrane technology, Kluwer Academic Publishers: Netherlands, 2003, p. 380-391.

2. Khan, J.; Tripathi, B. P.; Saxena, A.; Shahi, V. K.; Electrochim. Acta 2007, 52, 6719 .

3. Kendall, A. I.; Gebauer-Fuelnegg, E.; .J. Chem. Educ. 1931, 8, 1634.

4. García-García, V.; Montiel, V.; González-García, J.; Exposito, E.; Iniesta, J.; Bonete, P.; Inglés, M.; J. Chem. Educ. 2000, 77, 1477.

5. Farrell, S.; Hesketh, R. P.; Slater, C. S.; Chem. Eng. Ed. 2003, 37, 52.

6. Farrell, S.; Hesketh, R. P.; Savelski, M. J.; Chem. Eng. Ed. 2003, 37, 68.

7. Elminadaoui, A.; Elhannouni, F.; Menkouchi Salí, M. A.; Chay, L.; Elabbassi, H.; Hafsi, M.; Largeteau, D.; Dasalination 2001, 136, 325.

8. Ariza, M. J.; Otero, T. F.; J. Membr. Sci. 2007, 290, 241.

9. Chuanhui, H.; Tongwen X.; Yaping, Z.; Yanhong, X.; Guangwen, C.; J. Membr. Sci. 2007, 288, 1.

10. Xu, T.; J. Membr. Sci. 2005, 263, 1 .

11. Jayshree, R.; Tulsi, M.; Talanta 2007, 71, 1054.

12. Cowan, D.; Brown, J.; Ind. Eng. Chem. 1959, 51, 1445. 


\section{CONSTRUCCION Y UTILIZACION DE UN ELECTRODIALIZADOR EN SERIE PARA LABORATORIO}

\section{Curvas de polarización}

La Figura 1S esquematiza una curva de polarización típica en la que ocurren cada uno de los procesos mencionados durante el trasporte iónico.

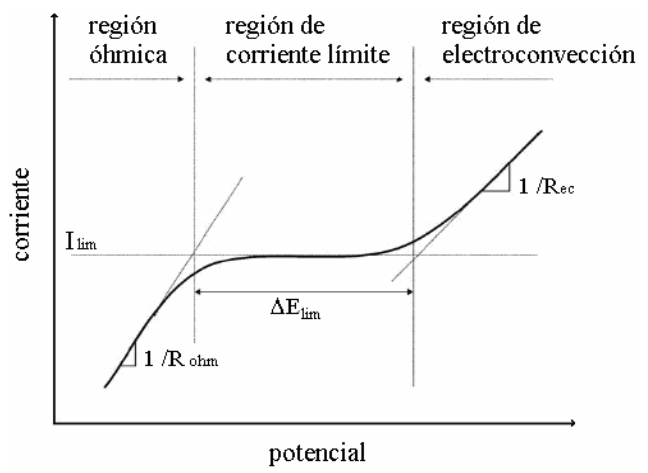

Figura 1S. Esquema de una curva típica de polarización experimental en donde se aprecian las tres zonas características: Región ohmica, región de corriente límite y región de electroconvección

Una curva de polarización típica posee tres regiones características:

1. Región óhmica. A bajas densidades de corriente se tiene una relación lineal entre la corriente y el potencial que se desarrolla en la membrana durante la migración de los contrapones a través de ella. El proceso esta gobernado por la ley de Ohm y muestra que cuando la densidad de corriente se incrementa la concentración del contraión en la capa de difusión del diluido disminuye y la resistencia se incrementa.

2. Región de corriente límite. En esta zona se observa una meseta ya que la corriente permanece casi constante aún con los incrementos de potencial. En esta región se inicia la electrólisis del agua generándose nuevas especies $\left(\mathrm{H}^{+}\right.$y $\left.\mathrm{OH}^{-}\right)$que serán las encargadas de transportar la carga en solución, disminuyendo la eficiencia de la corriente. La frontera entre estas regiones indica el valor de la corriente límite, $i_{\text {lim }}\left(\mathrm{o} \mathrm{I}_{\mathrm{lim}}\right)$ el cual puede ser obtenido por el método de las tangentes puesto que ocurre un cambio de pendiente marcado o por el método de Cowan y colaboradores. ${ }^{1}$

3. Región de electroconvección. El aumento de la corriente ocurre nuevamente y puede deberse a diferentes razones. Entre las diferentes teorías que explican este efecto, la propuesta por Rubins-

*e-mail: antonio.montes@uaslp.mx tein y colaboradores ${ }^{2,3}$ parece más realista ya que considera que las cargas fijas no están distribuidas homogéneamente en la superficie de la membrana por lo que la interacción con el campo eléctrico desestabiliza la capa de difusión destruyéndola, reactivando así el transporte de las especies iónicas en esas regiones, y por consecuencia el aumento de la corriente.

Algunos ejemplos de curvas de polarización obtenidas con este dispositivo se muestran en la Figura 2S.

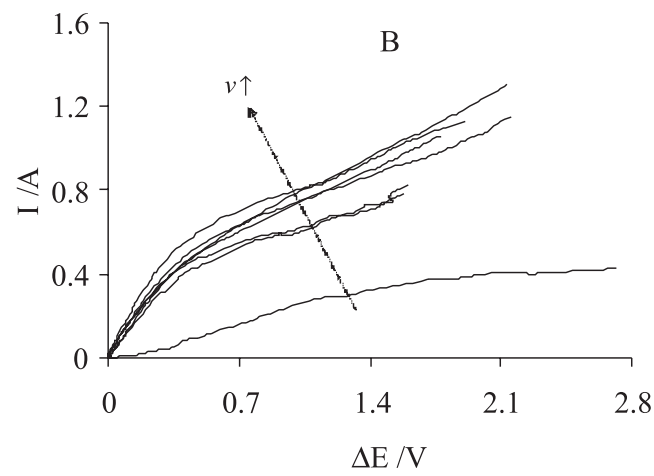

Figura 2S. Curvas de polarización para la membrana ACS a diferentes velocidades de alimentación (v: 0, 2,5, 5, 10, 15, 25, $35 \mathrm{~mL} \mathrm{~min}^{-1}$ ) a 0,1 M de nitratos

\section{Curvas de Cowan para determinar la $i_{\lim }$}

Este método considera que el voltaje que pasa por una celda de electrodiálsis (V) consta de diferentes términos de potencial entre los que se encuentran el potencial en los electrodos $\mathrm{V}_{\mathrm{e}}$, el potencial debido a la polarización por concentración $\mathrm{V}_{\mathrm{c}}$, el potencial por polarización $\mathrm{V}_{\mathrm{p}}$ y los términos óhmicos $\mathrm{IR}_{\mathrm{ohm}}$ :

$\mathrm{V}=\mathrm{IR}_{\mathrm{ohm}}+\mathrm{V}_{\mathrm{e}}+\mathrm{V}_{\mathrm{c}}+\mathrm{V}_{\mathrm{p}}$

A partir de esta expresión se puede obtener la expresión siguiente al dividir entre la corriente que fluye en la celda:

$\mathrm{V} / \mathrm{I}=\mathrm{R}_{\mathrm{ohm}}+\frac{\left(\mathrm{V}_{\mathrm{e}}+\mathrm{V}_{\mathrm{c}}+\mathrm{V}_{\mathrm{p}}\right)}{\mathrm{I}}$

De acuerdo con Cowan y Brown ${ }^{1}$ se puede utilizar esta ecuación para obtener la curva de V/I en función del reciproco de la corriente 


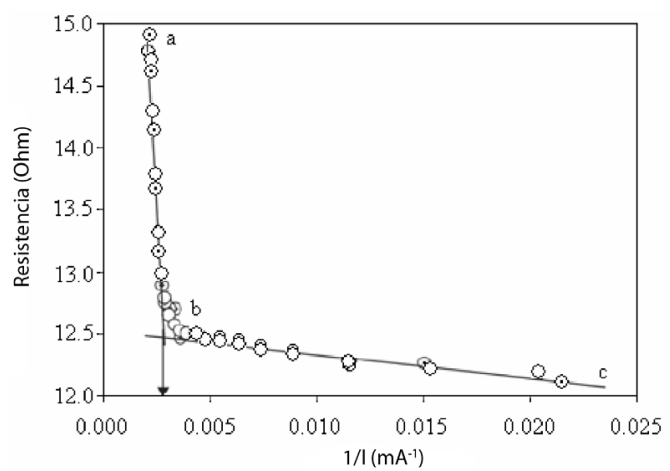

Figura 3S. Obtención de la corriente límite a partir de una curva de Cowan que fluye en la celda $\left(\mathrm{I}^{-1}\right)$ para obtener la corriente límite (Figura $3 \mathrm{~S})$.

Según estos autores la intercesión de las líneas a-b y b-c generan el valor de $i_{\text {lim }}\left(\right.$ o $\left.\mathrm{I}_{\text {lim }}\right)$.

\section{REFERENCIAS}

1. Cowan, D.; Brown, J.; Ind. Eng. Chem. 1959, 51, 1445.

2. Rubinstein, I.; Zaltaman, B.; Phys. Rev. E 2000, 62, 2238.

3. Balster, J.; Yildrim, M. H.; Stamatialis, D. F.; Ibanez, R. Lammertink, R. G. H.; Jordan, V.; Wessling, M.; J. Phys. Chem. B 2007, 111, 2152. 